تدريب ألعاب القوى للسيدات في ظل متغيرات المجتمع الفلسطيني.

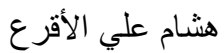

أستاذ التدريب الرياضي المشارك بكلية التربية البدنية والرياضة - جامعة الأقصى ـ فلسطين.

المقدمة واهمية البحث

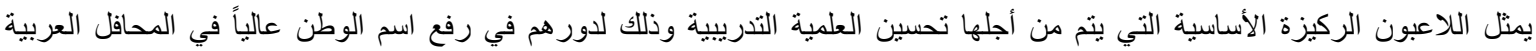

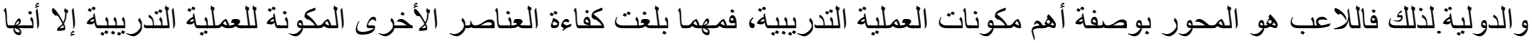
تظل محدودة التأثير إذا لم يوجد لاعب يتمتع بالاستقرار النفسي والأمن السياسي و الاجتماعي بالإضافة لتمتعه بالقدرات البدنية الخاصة بطبيعة الإنة

النشاط الرياضي.

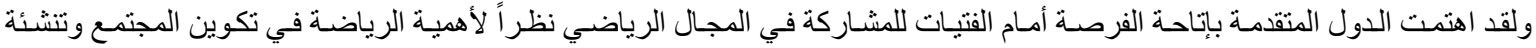

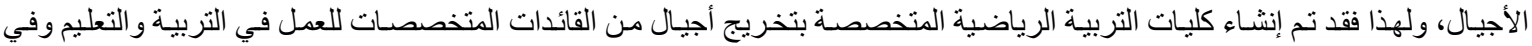
التدريب بالأنديـة بالإضـافة لإتاحـة الفرصـة لعدد كبير من الفتيات لممارسـة العديد من الأنثطة الرياضية في كافـة المستويات (عبد الخـالق:

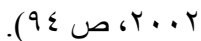

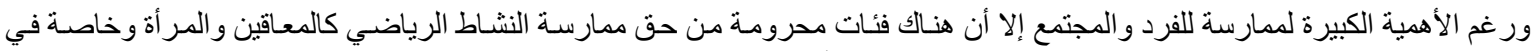

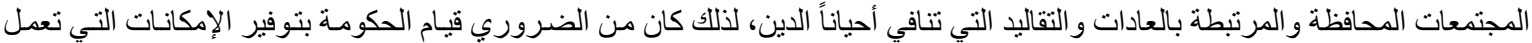

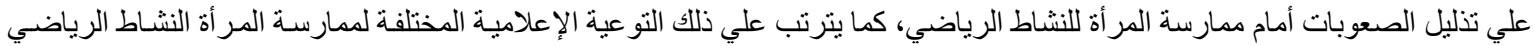

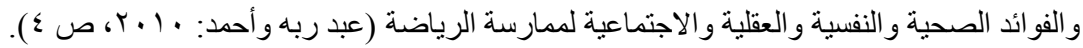

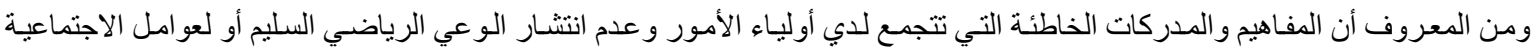

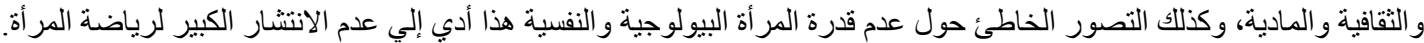

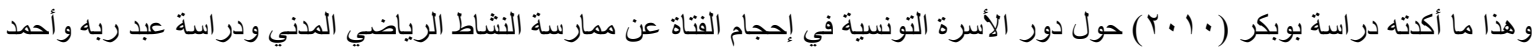

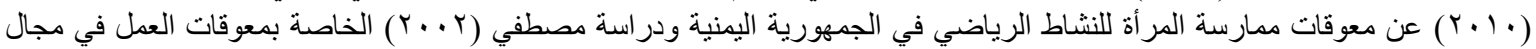

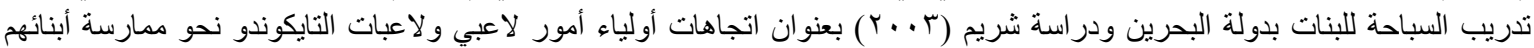

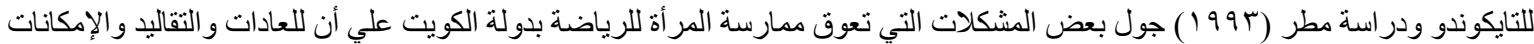

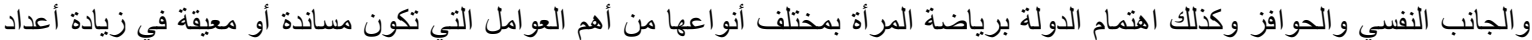
المشاركات في ممارسة الألعاب الرياضية المختلفة.

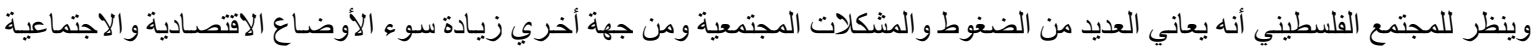
والأمنية و النفسية مما يعوق الممارسات الايجابية للانشطة الرياضية (محسن: V ... Y، ص V V ).

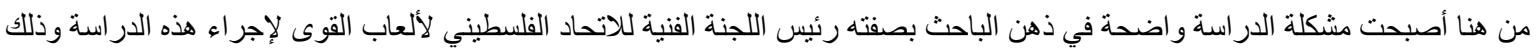

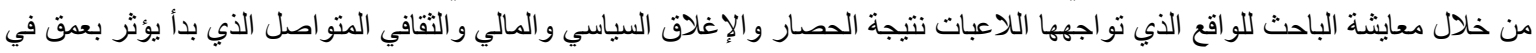

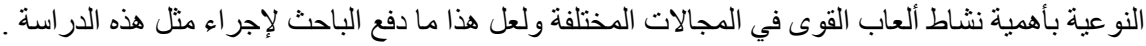

لذلك يري الباحث ضرورة التعرف علي جميع المتغيرات داخل المجتمع الفلسطيني و التني يمكن لها أن تؤثر إيجاباً أو سلباً علي تحقيق المستوي

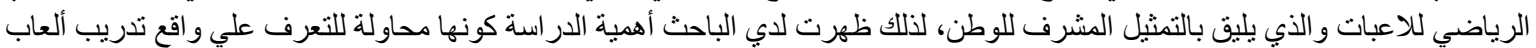

القوى للسيدات في ظل متغير ات الات المجتمع الفلسطيني.

مشكلة الاراسة:

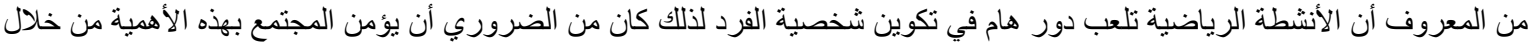

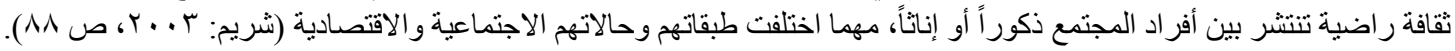

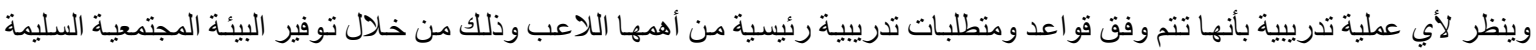

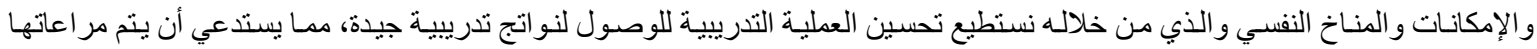

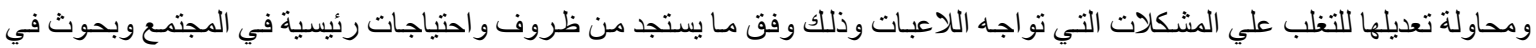

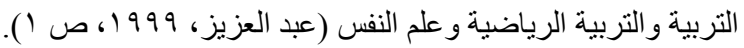




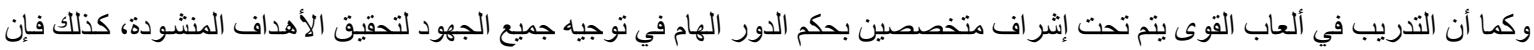

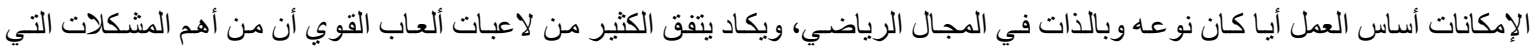

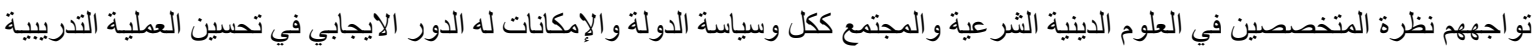
ومخرجاتها.

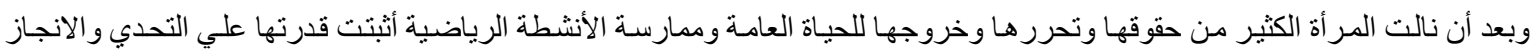

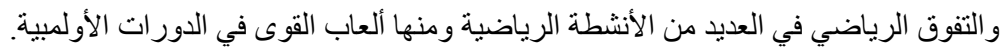
وبالر غم من أن المر أة نالت جز هـ حقوقها الرياضية إلا واجهت العديد من العو ائق المجتمعية المرتبطة بالعادات و التقاليد.

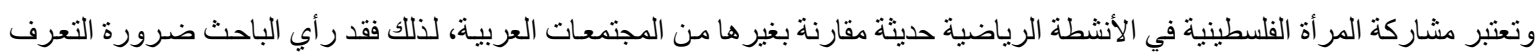

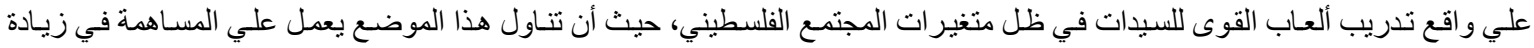
مشاركة وممارسة المر أة الفلسطينية للنشاط الرياضي مستقبلاً. وبإطلاع الباحث علي الدراسات في هذا المجال كدر اسة وجد ندرة في الأبحاث التي تناولت تدريب السيدات وبالذات التات في ألعاب القوى و علي

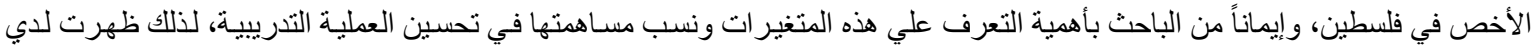

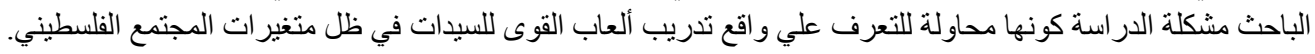

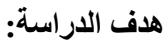
هدفت الدر اسة إلى التعرف على تدريب ألعاب القوى للسيدات في ظل متغير ات المجتمع الفلسطيني. تساؤل الاراسة: ما مستوى تدريب ألعاب القوى للسيدات في ظل متغير ات المجتمع الفلسطيني. مصطلح الاراسة:

- المتغيرات المجتمعية: هي "الأوضاع التي يعيثها المجتمع الفلسطيني وتثمل الجوانب السياسية "الأمنية" والاقتصادية و النفسية

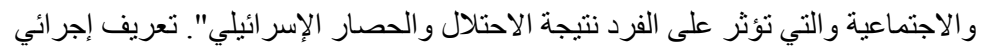

إجراعات الاراسة:

منهج الاراسة: استخدم الباحث المنهج الوصفي بالأسلوب المسحي لملائمته لطبيعة الدراسة.

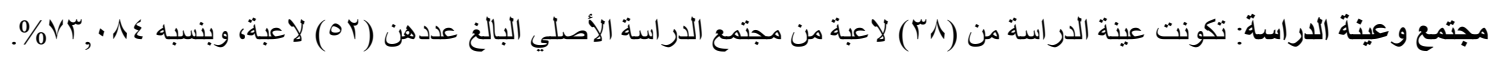
مجالات الدراسة:

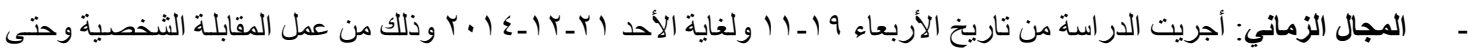
استخلاص النتائج.

- المجال الجغرافي: تم تطبيق الدراسة في فلسطين بمقر الاتحاد الفلسطيني باللجنة الأولمبية و أماكن التدريب بالمحافظات الثمالية

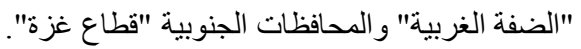

أدوات جمع البيانات:

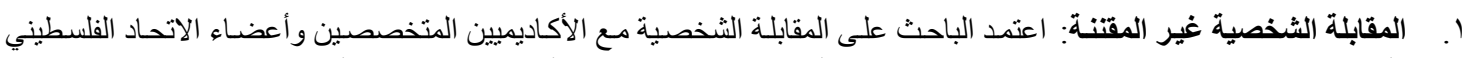

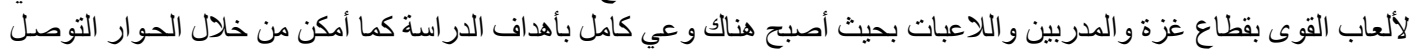
للمحاور الأساسية لاستمارة الاستبيان. r. استمارة استبيان تدريب ألعاب القوى للسيدات في ظل متغير ات الهتمع الفلسطيني " إعداد الباحث" ملحق "ب" و اتبعت الباحث في إعداده الخطوات التالية:

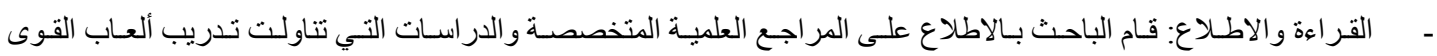
والمتغير ات المجتمعية الفلسطينية بهدف تحديد مضمون فقر الباح الات محاور الاستبيان. - - تحديد الهدف من الاستمارة: تم تحديد هدف استمارة الاستبيان بالتعرف علي و اقع تدريب ألعاب القوى للسيدات في ظل متغيرات المجتمع الفلسطيني. 
تحديد محاور الاستمارة: بعد الاطلاع علي الدراسات والبحوث العلمية وتحديد هدف الاستمارة ومن خـلال المقابلات الثخصية

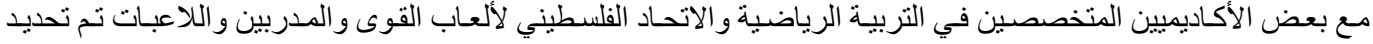
المحاور الأساسية للدر اسة وتم عرض هذه المحاور على عدد (V) خبر اء يحملون درجة الدكتور اهوسنوات خبرة في التدريس لا

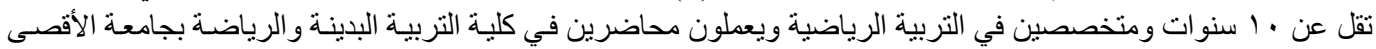

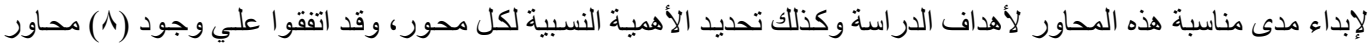
للاستمارة، وفيما يلي عرض للمحاور والنسب المئوية لأر اء الخبر اء كما يوضحه الجدول التالي:

جدول (1)

أراء الخبراء لمحاور الاستمارة قبل وبعد التحكيم والنسب المئوية

\begin{tabular}{|c|c|c|c|}
\hline \multicolumn{2}{|c|}{ أعضاء هيئة التدريس } & \multirow{2}{*}{ المحاور } & \multirow{2}{*}{ s } \\
\hline النسبة المئوية & تكرار الموافقة & & \\
\hline$\% 1 \ldots$ & $v$ & المحور الديني & $T$ \\
\hline$\% 1 \ldots$ & v & المحور المجتمعي & $r$ \\
\hline$\% 1 \ldots$ & V & المحور الأسري & $r$ \\
\hline$\% 1 \ldots$ & V & المحور الإعلامي & $\varepsilon$ \\
\hline$\% 1 \ldots$ & V & المحور السياسي & 0 \\
\hline$\% 1 \ldots$ & V & المحور النفسي & 7 \\
\hline$\% 1 \ldots$ & V & محور الإمكانات & V \\
\hline$\% 1 \ldots$ & $\mathrm{V}$ & المحور الإداري & $\Lambda$ \\
\hline
\end{tabular}

يتضح من الجدول رقم ( 1 ) أن جميع المحاور الخاصة بالاستمارة قد حصلت على نسبة ( · (1\%) وقد تم قبول تلك المحاور.

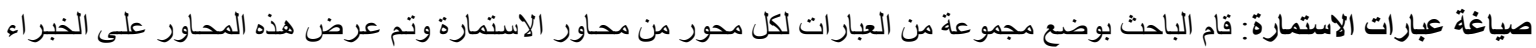

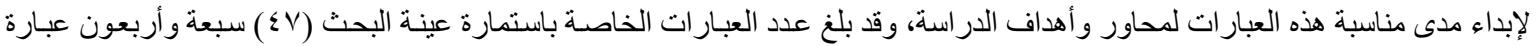

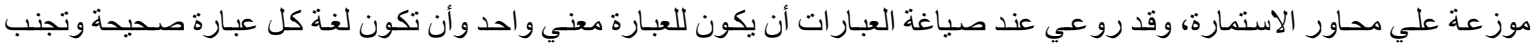

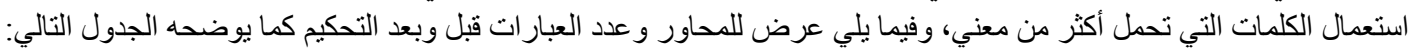

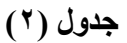

المحاور وعدد العبارات قبل وبعد التحكيم الخاصة باستمارة الاستبيان

\begin{tabular}{|c|c|c|c|}
\hline \multicolumn{2}{|c|}{ العبارات } & \multirow{2}{*}{ المحاور } & \multirow[b]{2}{*}{ b } \\
\hline بعد التحكيم & العبارات قبل التحكيم & & \\
\hline 7 & 0 & المحور الديني & 1 \\
\hline V & $\Lambda$ & المحور المجتمعي & r \\
\hline 7 & 7 & المحور الأسري & $r$ \\
\hline 0 & 7 & المحور الإعلامي & $\varepsilon$ \\
\hline 7 & $\wedge$ & المحور السياسي & 0 \\
\hline 0 & V & المحور النفسي & 7 \\
\hline 7 & 9 & محور الإمكانات & V \\
\hline 7 & $\mathrm{~V}$ & المحور الإداري & $\Lambda$ \\
\hline$\varepsilon V$ & 07 & سئلة الاستمارة & \\
\hline
\end{tabular}

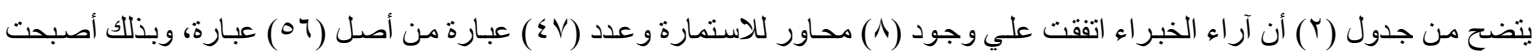

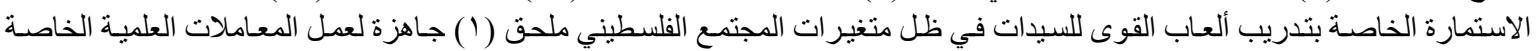

تصحيح الاستمارة: لتصحيح الاستمارة قام الباحث بوضع ميزان تقديري ثلاثي، وقد تم تصحيح العبارات كالتالي:

\begin{tabular}{|c|c|c|c|c|}
\hline \multicolumn{2}{|c|}{ العبارات السلبية } & \multicolumn{2}{|c|}{ العبارات الإيجابية } & p \\
\hline درجة & نعم & ثلاث درجات & نعم & 1 \\
\hline درجتان & إلي حد ما & درجتان & إلي حد ما & $r$ \\
\hline ثلاث درجات & $\gamma$ & درجة & $\gamma$ & $r$ \\
\hline
\end{tabular}


المعاملات العلمية للاستمارة:

أولاً: صدق الاستمارة

قام الباحث باحتساب صدق الاستمارة باستخدام الطرق التالية:

ا. . صدق المحتوي: قام الباحث بإيجاد صدق الاستمارة عن طريق صدق المحكمين وذللك بعرض استمارة الاستمارة في صورتها

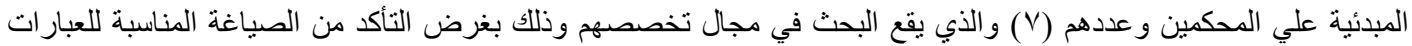

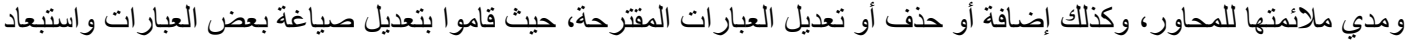

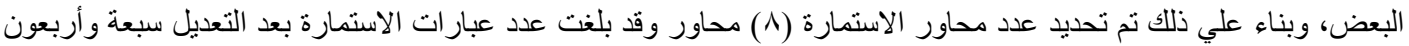

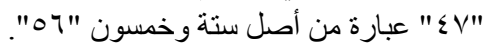

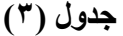

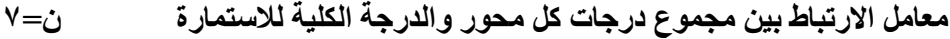

\begin{tabular}{|c|c|c|}
\hline معامل الارتباط & المحاور & م \\
\hline$\cdot .9 \cdot r$ & المحور الديني & 1 \\
\hline $.91 r$ & المحور المجتمعي & r \\
\hline$\cdot . \wedge \vee q$ & المحور الأسري & r \\
\hline$\because \wedge .0$ & المحور الإعلامي & $\varepsilon$ \\
\hline $.9 \pi$ & المحور السياسي & 0 \\
\hline$\cdot \wedge \leq Y$ & المحور النفسي & 7 \\
\hline.$\wedge 01$ & محور الإمكانات & V \\
\hline .149 & المحور الإداري & $\wedge$ \\
\hline
\end{tabular}

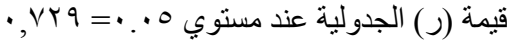

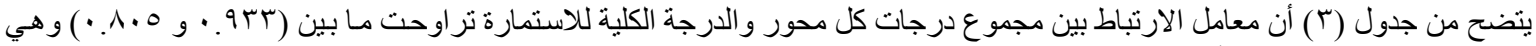

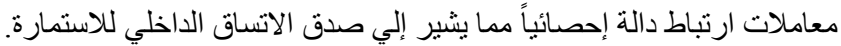

ثانياً: ثبات الاستمارة

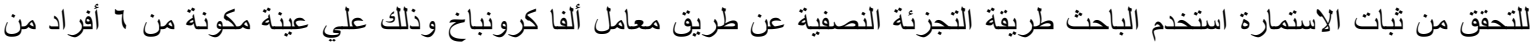

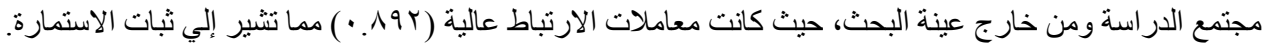

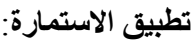

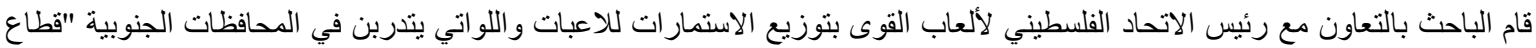
غزة"، وكذلك بإرسال باقي الاستمار ات إلي اللاعبات في المحافظات الثمالية "الضفة الغربية" بواسطة الاتحات الاتحاد بالبريد الإلكتروني.

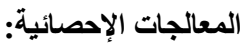

$$
\begin{aligned}
& \text { ا - مجموع الاستجابات. } \\
& \text { r - المتوسط الحسابي. } \\
& \text { ب- النسب المئوية. } \\
& \text { ع - ارتباط بيرسون. }
\end{aligned}
$$

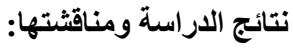

نص السؤ ال الرئيسي على: ما مستوى تدريب ألعاب القوى للسيدات في ظل متغيرات المجتمع الفلسطيني. وللإجابة عن السؤ ال قام الباحث بايجاد المنوسط الحسابي والوزن النسبي لكل محور من محاور الاستبيان والجداول التالية توضح ذلك. 


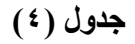

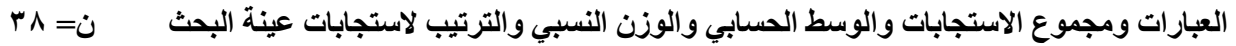

\begin{tabular}{|c|c|c|c|c|}
\hline الترتيب & الوزن النسبي & المتوسط الحسابي & مجموع الاستجابات & رقم الفقرة \\
\hline IT & V9.7V & r.rq & 91 & 1 \\
\hline TY & TV.TV & $r . r$ & VV & T \\
\hline 0 & AV.TV & T.7T & $1 \ldots$ & $r$ \\
\hline IV & VY.TV & r.ru & $\Lambda \varepsilon$ & $\varepsilon$ \\
\hline$\varepsilon r$ & $\leqslant \varepsilon$ & $1 . \mu Y$ & 0. & 0 \\
\hline$r$. & VI & T.IT & NI & 7 \\
\hline ro & 01 & 1.04 & 01 & $\mathrm{~V}$ \\
\hline $1 \pi$ & 89 & T.TV & 9. & $\Lambda$ \\
\hline Tr & $0 \leqslant . T T$ & $1.7 \pi$ & $\pi r$ & 9 \\
\hline$\varepsilon$. & $\varepsilon V, r \mu$ & $1 . \leqslant Y$ & $0 \xi$ & 1. \\
\hline rA & $\pi$ & 1.19 & VY & 11 \\
\hline TY & TV.TV & $r . \cdot r$ & VV & ir \\
\hline$\varepsilon$. & $\varepsilon V . r T$ & $1 . \varepsilon Y$ & $0 \leqslant$ & 14 \\
\hline YI & $\pi \wedge . r \mu$ & r.. & $\vee \wedge$ & $1 \varepsilon$ \\
\hline T & 0. & 1.0 & OV & 10 \\
\hline 7 & AE.TY & r.or & 97 & 17 \\
\hline$r$. & rו. & $1 . \wedge \varepsilon$ & $V \cdot$ & IV \\
\hline$V$ & זr.r. & T. $\leqslant V$ & $9 \leqslant$ & 11 \\
\hline$r$ & 94 & T.Vq & 1.7 & 19 \\
\hline rq & אז.rT & $1 . \wedge V$ & 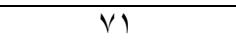 & $r$. \\
\hline$\leqslant 0$ & $\varepsilon Y$ & 1.17 & $\sum \Lambda$ & YI \\
\hline$V$ & AT.rT & T. $\leqslant V$ & $9 \xi$ & TY \\
\hline$r q$ & $\leq 9$ & $1 . \leqslant V$ & 07 & Tr \\
\hline$\varepsilon r$ & $\leqslant 0.7 V$ & $1 . r V$ & or & $T \leq$ \\
\hline 11 & NI.TV & T. $\leqslant 0$ & 94 & To \\
\hline IV & Vr.TV & r.r. & $\Lambda \varepsilon$ & YT \\
\hline$V$ & Ar.rT & $r . \leqslant V$ & $9 \leqslant$ & TV \\
\hline$\varepsilon \varepsilon$ & $\varepsilon r$ & 1.19 & $\sum 9$ & TA \\
\hline TI & 07 & 1.71 & $7 \varepsilon$ & rq \\
\hline 17 & VO.rT & Y.YT & $\Lambda 7$ & $r$. \\
\hline$\leqslant 0$ & $\varepsilon Y$ & 1.17 & $\sum \wedge$ & r \\
\hline V & גז.rT & Y. $\leqslant V$ & $9 \varepsilon$ & TY \\
\hline 1 & 1 & $r$ & $11 \varepsilon$ & rr \\
\hline 19 & $V Y$ & T.17 & $\Delta r$ & $\Gamma \varepsilon$ \\
\hline$r$ & $9 \varepsilon$ & T.AY & $1 \cdot V$ & ro \\
\hline TV & 70 & 1.90 & $V \varepsilon$ & $r 4$ \\
\hline r & 07 & 1.71 & $7 \varepsilon$ & $T V$ \\
\hline Tr & $00 . \mu T$ & 1.77 & 74 & rA \\
\hline $1 \pi$ & 19 & T.TV & 9. & $r q$ \\
\hline 10 & VV.rT & T.MT & $\Lambda \Lambda$ & $\varepsilon$. \\
\hline Tr & $0 \leqslant . M T$ & 1.74 & 74 & §1 \\
\hline TO & $70.7 \mathrm{~V}$ & $1.9 \mathrm{~V}$ & Vo & $\sum Y$ \\
\hline$r$ & 94 & r.v9 & 1.7 & $\varepsilon r$ \\
\hline To & $70.7 \mathrm{~V}$ & $1.9 \mathrm{~V}$ & Vo & $\varepsilon \varepsilon$ \\
\hline$\leqslant 0$ & $\varepsilon r$ & $1 . Y 7$ & $\varepsilon \wedge$ & $\leqslant 0$ \\
\hline YY & TV.TV & $r . r$ & VV & $\sum 7$ \\
\hline rד & 0 & 1.0 & OV & $\sum V$ \\
\hline
\end{tabular}


المحور الأول: الايني

جدول (•)

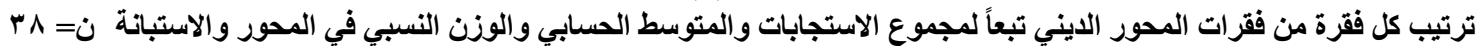

\begin{tabular}{|c|c|c|c|c|c|c|}
\hline الترتيب في المحور & الاستببانة في & الوزن النسبي & المتوسط الحسابي & مجموع الاستجابات & رقم الفقرة & م \\
\hline$r$ & Ir & V9.TV & Y.rq & 91 & 1 & 1 \\
\hline 0 & Tr & سז.r. & $1.7 \pi$ & $\pi r$ & 9 & $r$ \\
\hline$\varepsilon$ & $r$. & זr. & $1 . \wedge \varepsilon$ & V. & IV & $r$ \\
\hline$r$ & 11 & NI.TV & $r_{.} \leqslant 0$ & 94 & ro & $\varepsilon$ \\
\hline 1 & 1 & $1 \ldots$ & $r$ & $11 \leqslant$ & Tr & 0 \\
\hline \multirow[t]{2}{*}{0} & rr & O & 1.74 & $T$ & $\leqslant 1$ & 7 \\
\hline & & VI.19 & 4.17 & 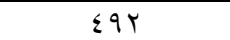 & \multicolumn{2}{|c|}{ المجموع } \\
\hline
\end{tabular}

يتضح من الجدول (0) و الخاص بمجموع الاستجابات و المتوسط الحسابي لاستجابات عينة الدراسة على كل فقرة من فقرات المحور الديني

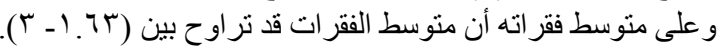

المحور الثاني: المجتمعي

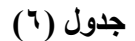

ترتيب كل فقرة من فقرات المحور المجتمعي تبعاً لمجموع الاستجابات والمتوسط الحسابي والوزن النسبي في المحور والاستبانة ن= ^r

\begin{tabular}{|c|c|c|c|c|c|c|}
\hline الترتيب في المحور & الترتيب في الاستبانة & الوزن النسبي & المتوسط الحسابي & مجموع الاستجابات & رقم الفقرة & م \\
\hline$\varepsilon$ & rr & TV.TV & $r . r$ & $V V$ & $r$ & 1 \\
\hline V & $\varepsilon$. & $\varepsilon V, r r$ & $1 . \leqslant r$ & $0 \leqslant$ & 1. & $r$ \\
\hline 1 & V & הT.rT & $r . \varepsilon V$ & $9 \varepsilon$ & 11 & $r$ \\
\hline$r$ & IV & VT.TV & $r . r_{1}$ & $\wedge \varepsilon$ & rT & $\varepsilon$ \\
\hline$r$ & 19 & VY & T.17 & Ar & $\Gamma \varepsilon$ & 0 \\
\hline 0 & To & $70.7 \mathrm{~V}$ & $1.9 \mathrm{~V}$ & vo & $\varepsilon r$ & 7 \\
\hline \multirow[t]{2}{*}{7} & rq & 0. & 1.0 & ov & $\varepsilon V$ & v \\
\hline & & 70.04 & $1.9 V$ & OrT & \multicolumn{2}{|c|}{ المجمو ع } \\
\hline
\end{tabular}

يتضح من الجدول (7) و الخاص بمجموع الاستجابات و المنوسط الحسابي لاستجابات عينة الدراسة على كل فقرة من فقرات المحور المجتمعي

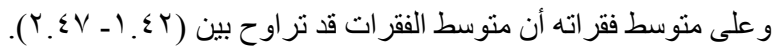

المحور الثالث: الأسري

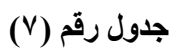

ترتيب كل فقرة من فقرات المحور الأسري تبعاً لمجموع الاستجابات و المتوسط الحسابي والوزن النسبي في المحور والاستبانةن= ^

\begin{tabular}{|c|c|c|c|c|c|c|}
\hline الترتيب في المحور & الترتيب في الاستبانة & الوزن النسبي & المتوسط الحسابي & مجموع الاستجابات & رقم الفقرة & م \\
\hline$\varepsilon$ & 0 & AV.TV & r.Tr & $1 \ldots$ & $r$ & 1 \\
\hline 7 & $r \wedge$ & תוד & 1.19 & $V Y$ & 11 & $r$ \\
\hline r & $r$ & $9 \pi$ & r.vq & 1.7 & 19 & $r$ \\
\hline 0 & V & AY.rT & $r . \leqslant V$ & $9 \varepsilon$ & TV & $\varepsilon$ \\
\hline 1 & r & $9 \leqslant$ & r.Ar & $1 . V$ & ro & 0 \\
\hline \multirow[t]{2}{*}{ r } & $r$ & $9 \pi$ & r.vq & 1.7 & $\varepsilon r$ & 7 \\
\hline & & 10.r & Y.OV & 010 & \multicolumn{2}{|c|}{ المجمو ع } \\
\hline
\end{tabular}


يتضح من الجدول (V) و الخاص بمجموع الاستجابات والمتوسط الحسابي لاستجابات عينة الدراسة على كل فقرة من فقرات المحور الأسري

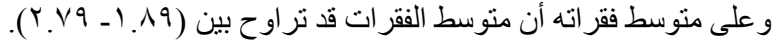

المحور الرابع: الإعلامي

جدول (^)

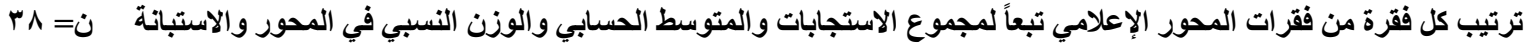

\begin{tabular}{|c|c|c|c|c|c|c|}
\hline الترتيب في المحور & الترتيب في الاستبانة & الوزن النسبي & المتوسط الحسابي & مجموع الاستجابات & رقم الفقرة & م \\
\hline 1 & IV & VT.TV & Y.Y & $\wedge \varepsilon$ & $\varepsilon$ & 1 \\
\hline r & rr & TV.TV & $r . r$ & VV & ir & r \\
\hline$\varepsilon$ & rq & אזיז.T & I.AV & VI & $r$. & r \\
\hline 0 & $\varepsilon \leq$ & $\varepsilon r$ & 1.49 & $\leqslant 9$ & rA & $\varepsilon$ \\
\hline$r$ & $r V$ & 70 & 1.90 & $V \varepsilon$ & r & 0 \\
\hline & & אוז.זד & I.AV & roo & \multicolumn{2}{|c|}{ المجموع } \\
\hline
\end{tabular}

يتضح من الجدول (^) و الخاص بمجموع الاستجابات و المتوسط الحسابي لاستجابات عينة الدراسة على كل فقرة من فقرات المحور الإعلامي

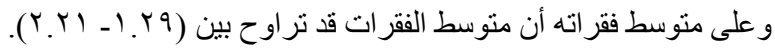
المحور الخامس: السياسي

\section{جدول (9)}

ترتيب كل فقرة من فقرات المحور السياسي تبعاً لمجموع الاستجابات والمتوسط الحسابي والوزن النسبي في المحور والاستبانة ن= ^r

\begin{tabular}{|c|c|c|c|c|c|c|}
\hline الترتيب في المحور & الترتيب في الاستبانة & الوزن النسبي & المتوسط الحسابي & مجموع الاستجابات & رقم الفقرة & م \\
\hline 0 & $\varepsilon r$ & $\varepsilon \varepsilon$ & Tr. & 0. & 0 & 1 \\
\hline$\varepsilon$ & $\varepsilon$. & זע.r & $1 . \leqslant Y$ & $0 \leqslant$ & Ir & r \\
\hline 7 & $\leqslant 0$ & $\varepsilon Y$ & 1.Y & $\leqslant \wedge$ & YI & r \\
\hline r & I & 07 & 1.71 & $7 \leq$ & rq & $\varepsilon$ \\
\hline r & ו & 07 & 1.71 & $7 \varepsilon$ & rV & 0 \\
\hline 1 & ro & $70.7 \mathrm{~V}$ & $1.9 V$ & Vo & $\varepsilon \varepsilon$ & 7 \\
\hline & & $01 . \lambda r$ & 1.07 & roo & \multicolumn{2}{|c|}{ المجموع } \\
\hline
\end{tabular}

يتضح من الجدول (9) و الخاص بمجموع الاستجابات والمتوسط الحسابي لاستجابات عينة الدراسة على كل فقرة من فقرات المحور السياسي

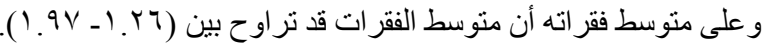
المحور السادس: النفسي

\section{جذول (· )}

ترتيب كل فقرة من فقرات المحور النفسي تبعاً لمجموع الاستجابات والمتوسط الحسابي والوزن النسبي في المحور والاستبانة ن=

\begin{tabular}{|c|c|c|c|c|c|c|}
\hline الترتيب في المحور & الترتيب في الاستبانة & الوزن النسبي & المتوسط الحسابي & مجموع الاستجابات & رقم الفقرة & م \\
\hline r & r. & V) & r. & $\wedge 1$ & 7 & 1 \\
\hline$\varepsilon$ & YI & (1.r. & $r .0$ & $\vee \wedge$ & $1 \varepsilon$ & r \\
\hline 1 & V & מז.ru & $r . \leqslant V$ & $9 \leq$ & rt & r \\
\hline r & 17 & vo.ru & דיtr & $\wedge T$ & r. & $\varepsilon$ \\
\hline \multirow[t]{2}{*}{0} & س & r.r. & 1.77 & 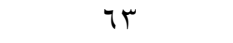 & rᄉ & 0 \\
\hline & & $V \cdot . \leqslant 7$ & r.11 & $\varepsilon \cdot r$ & \multicolumn{2}{|c|}{ المجموع } \\
\hline
\end{tabular}

يتضح من الجدول (• ( ) و الخاص بمجموع الاستجابات و المتوسط الحسابي لاستجابات عينة الدراسة على كل فقرة من فقرات المحور النفسي

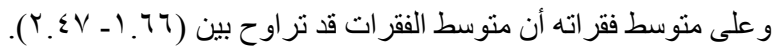


المحور السابع: الإمكانات

جدول (11)

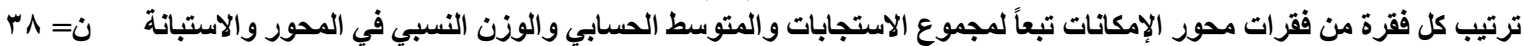

\begin{tabular}{|c|c|c|c|c|c|c|}
\hline الترتيب في المحور & الترتيب في الاستبانة & الوزن النسبي & المتوسط الحسابي & مجموع الاستجابات & رقم الفقرة & م \\
\hline$r$ & ro & 01 & $1.0 r$ & $0 \wedge$ & v & 1 \\
\hline r & ד & 0. & 1.0 & or & 10 & r \\
\hline$\varepsilon$ & rq & $\leqslant 9$ & $1 . \leqslant V$ & 07 & Tr & r \\
\hline 0 & $\leqslant 0$ & $\varepsilon r$ & $1 . Y 7$ & $\leqslant \wedge$ & r & $\varepsilon$ \\
\hline 1 & 14 & Vq & T.TV & 9. & rq & 0 \\
\hline \multirow[t]{2}{*}{0} & $\leq 0$ & $\varepsilon r$ & 1.17 & $\leqslant 1$ & $\leqslant 0$ & 7 \\
\hline & & Or.IV & $1.0 \mathrm{~V}$ & rov & \multicolumn{2}{|c|}{ المجموع } \\
\hline
\end{tabular}

يتضح من الجدول ( (1) و الخاص بمجموع الاستجابات و المتوسط الحسابي لاستجابات عينة الدر اسة على كل فقرة من فقر ات محور الإمكانات

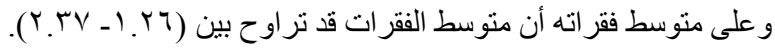
المحور الثامن: الإداري

جدول (11)

ترتيب كل فقرة من فقرات المحور الإداري تبعاً لمجموع الاستجابات والمتوسط الحسابي والوزن النسبي في المحور والاستبانة ن= ^

\begin{tabular}{|c|c|c|c|c|c|c|}
\hline الترتيب في المحور & الترتيب في الاستباتة & الوزن النسبي & المتوسط الحسابي & مجموع الاستجابات & رقم الفقرة & م \\
\hline$r$ & $1 \Gamma$ & $v q$ & r.rV & 9 & $\wedge$ & 1 \\
\hline 1 & 7 & As.rT & r.or & 97 & 17 & r \\
\hline 7 & $\varepsilon Y$ & $\leqslant 0.7 \mathrm{~V}$ & $1 . r v$ & or & $r \varepsilon$ & $r$ \\
\hline r & V & Ar.rT & $r . \leqslant V$ & $9 \leqslant$ & rr & $\varepsilon$ \\
\hline$\varepsilon$ & 10 & VV.TT & trut & 11 & $\varepsilon$. & 0 \\
\hline \multirow[t]{2}{*}{0} & KY & TV.TV & $r . r$ & VV & $\leq 7$ & 7 \\
\hline & & $V Y . V Y$ & $r .11$ & $\leq 9 V$ & \multicolumn{2}{|c|}{ المجموع } \\
\hline
\end{tabular}

يتضح من الجدول ( (1) و الخاص بمجموع الاستجابات والمتوسط الحسابي لاستجابات عينة الدراسة على كل فقرة من فقرات المحور الإداري

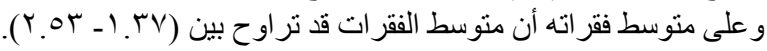

الأبعاد الكلية للقائمة:

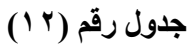

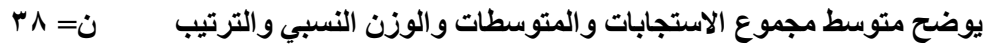

\begin{tabular}{|c|c|c|c|c|}
\hline الترتيب & الوزن النسبي & المتوسط الحسابي & مجموع الاستجابات & الأبعاد \\
\hline$r$ & $\vee 1.19$ & Y.17 & $\leq q r$ & المحور الديني \\
\hline 0 & 70.04 & $1.9 \mathrm{~V}$ & Orr & المحور المجتمعي \\
\hline 1 & 10.rr & Y.OV & 010 & المحور الأسري \\
\hline 7 & Tr.Tr & $1 . \wedge \mathrm{V}$ & $r 00$ & المحور الإعلامي \\
\hline$\wedge$ & 01.14 & 1.07 & $r 00$ & المحور السياسي \\
\hline$\varepsilon$ & $V \cdot . \leqslant 7$ & r.11 & $\varepsilon \cdot r$ & المحور النفسي \\
\hline V & Or.IV & $1.0 \mathrm{~V}$ & rov & محور الإمكانات \\
\hline \multirow[t]{2}{*}{ r } & VY.VY & r.11 & $\leq 9 V$ & المحور الإداري \\
\hline & 77.04 & r & ro7r & متوسط المجموع \\
\hline
\end{tabular}

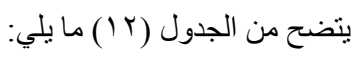


كانت النسبة المئوية للاستجابة الكلية لعينة اللاعبات متوسطة حيث بلغت (r.0r.7\%).

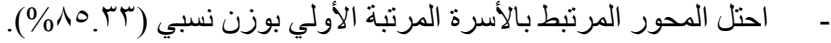

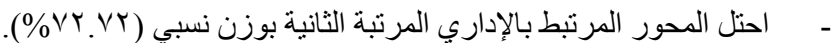

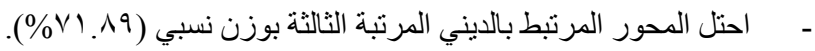

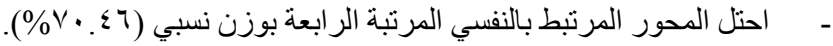

- احتل المحور المرتبط بالمجتمعي المرتبة الخامسة بوزن نسبي (ro.0r\%).

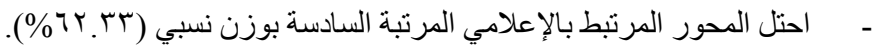

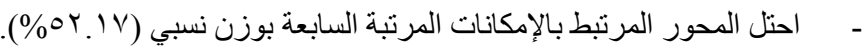

- احتل المحور المرتبط بالسياسي المرتبة الثامنة بوزن نسبي (r^. 10\%).

مناقشة النتائج:

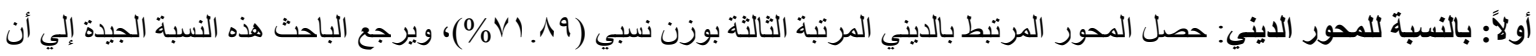

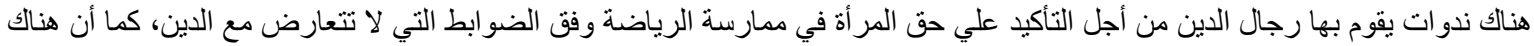

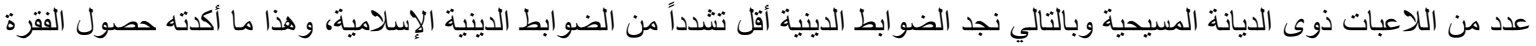

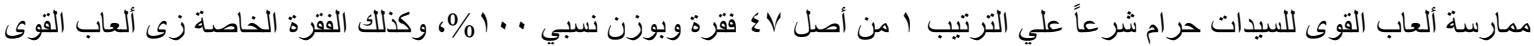

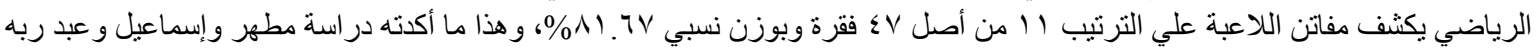

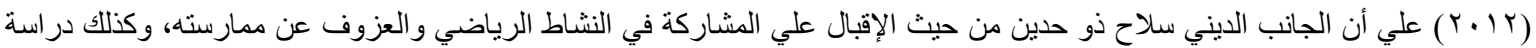

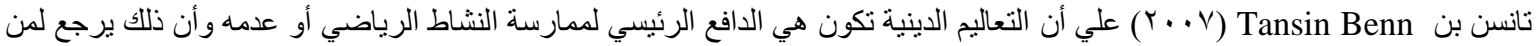

يقوم بنوضيح هذه الضو ابط الثر عية.

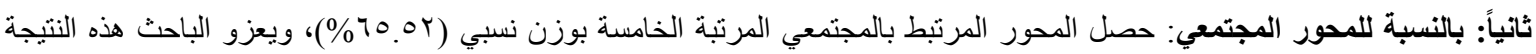

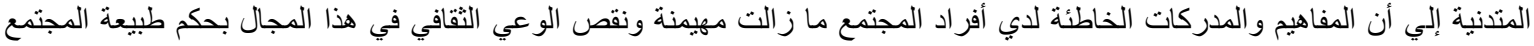

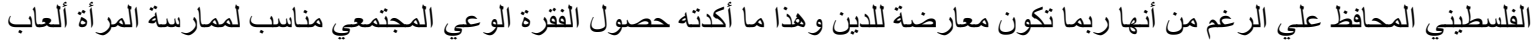

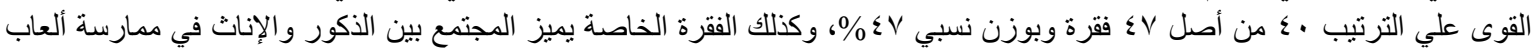

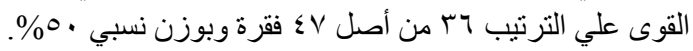

و هذه نتيجة طبيعية ومنطقية مقارنةً بأن النشاط الرياضي في فلسطين حديث جداً مقارنة بالدول العربية الثقيقة، كما أن عدم تو افر أندية وأماكن

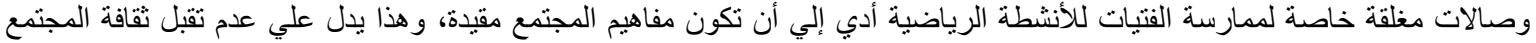

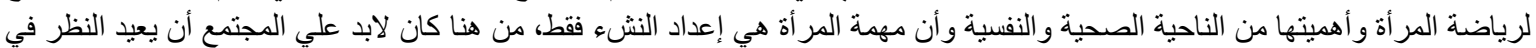
بعض العادات و التقاليد الخاطئة التي تمنع وتحد من مشاركتها في هذا النشاط و القتصاره علي الذكور.

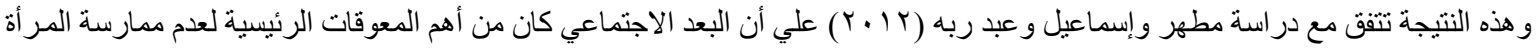

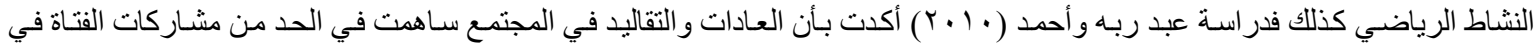

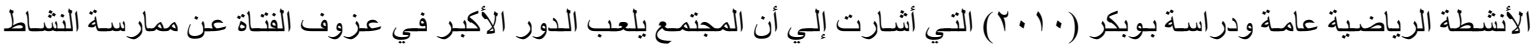

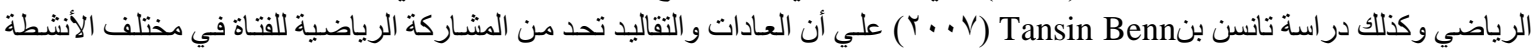
الداخلية والخارجية.

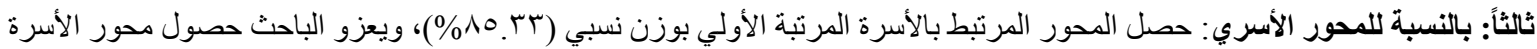

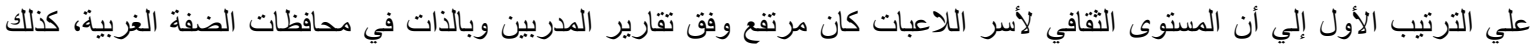

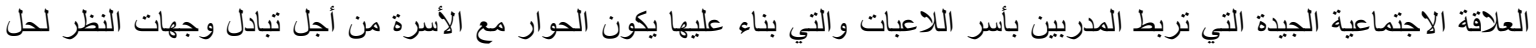

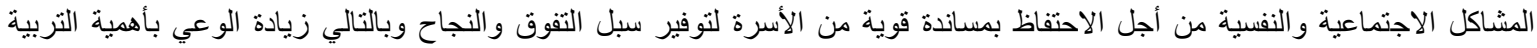

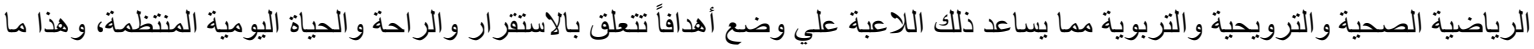

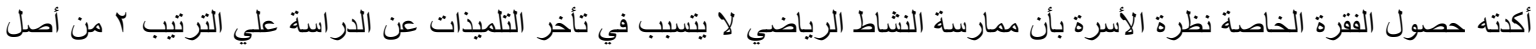

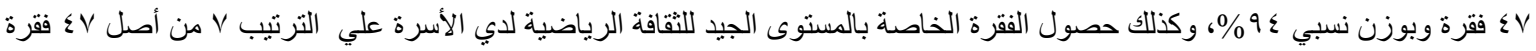

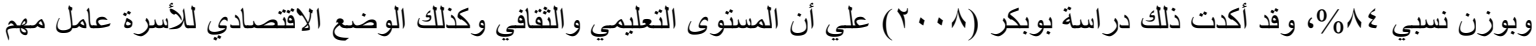

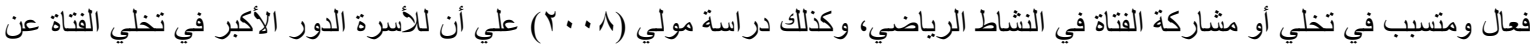
مشاركتها في الألعاب الرياضية. 


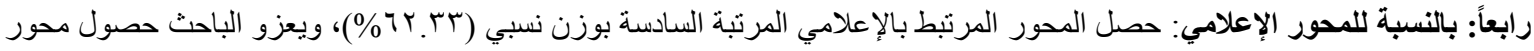

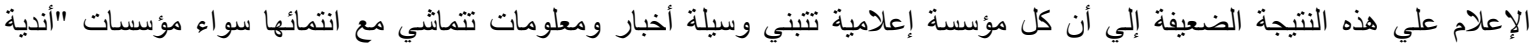

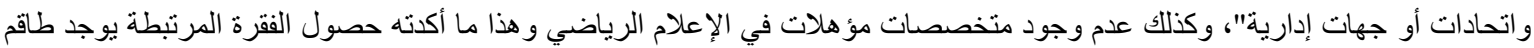

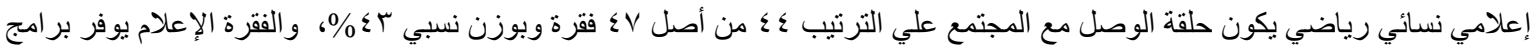

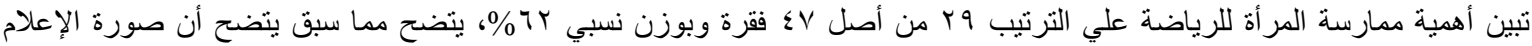

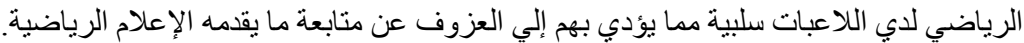

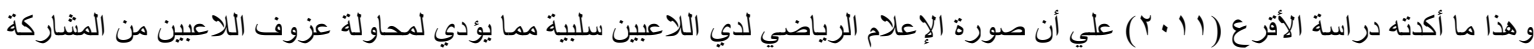

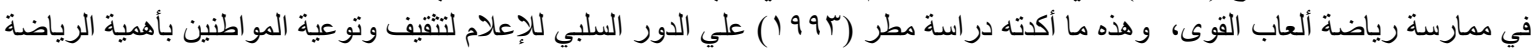
و ألعاب القوى ورفع مستوى الوعي الرياضي و الثقافة الرياضية بين أفراد المجتمع.

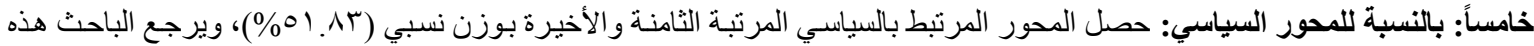

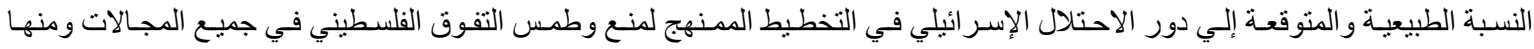

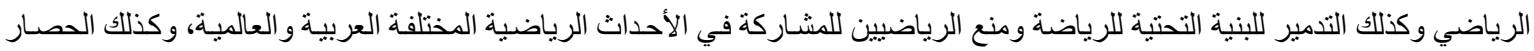

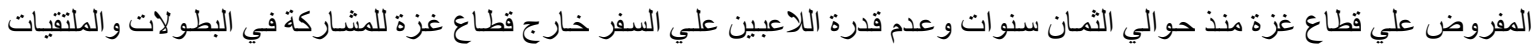

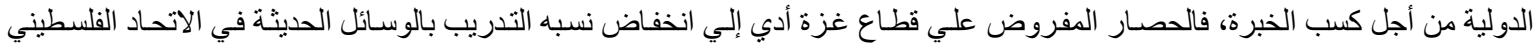

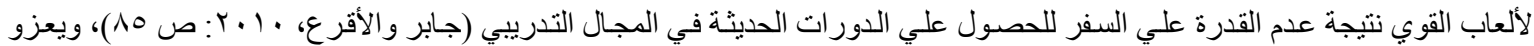

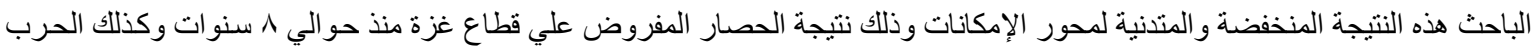

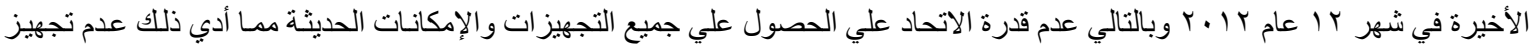

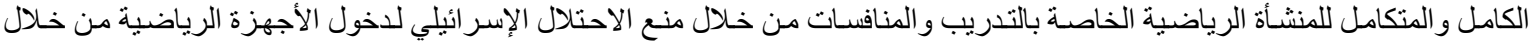

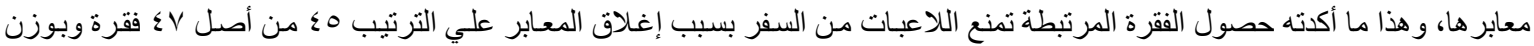

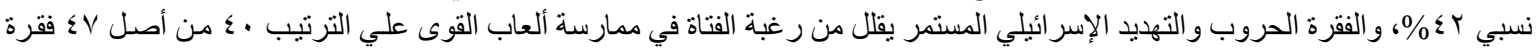

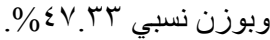

كما أن قلة الدعم المقدم من قبل الدولة يعمل وبشكل كبير علي زيادة الإقبال علي ممارسة رياضة ألعاب القوى و هذا ما أثنارت إليه دراسة

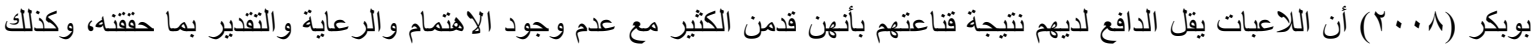

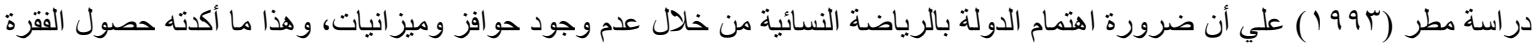

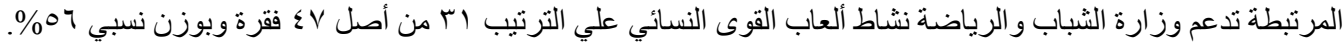

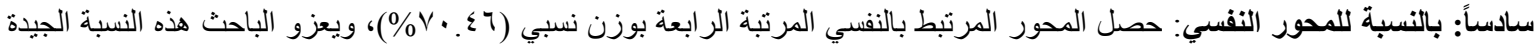

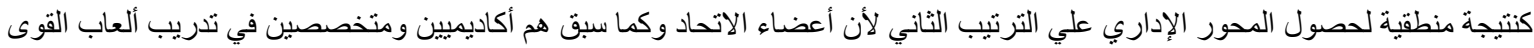

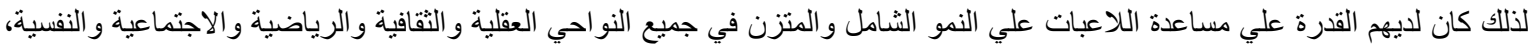

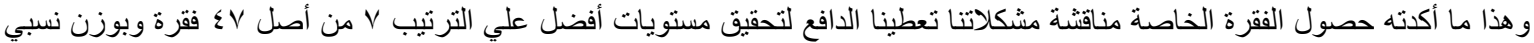

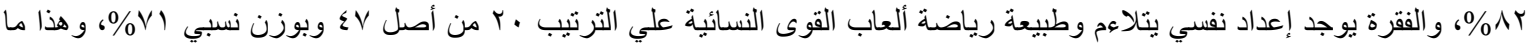

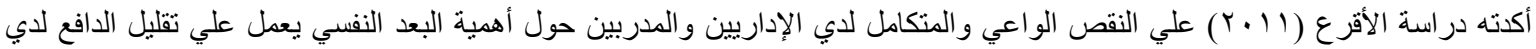

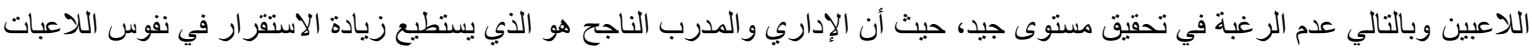
من أجل إعطاء التعليمات و النصائح و القرار ات للاعبين فئن بصورة سليمة.

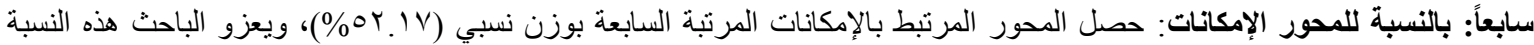

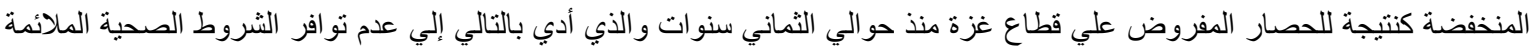

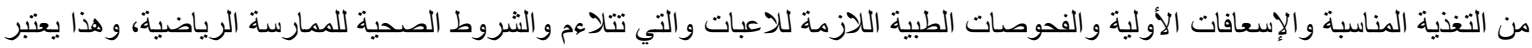

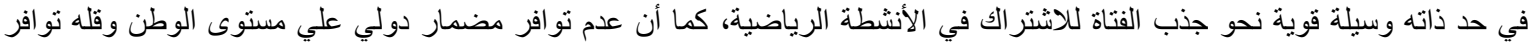

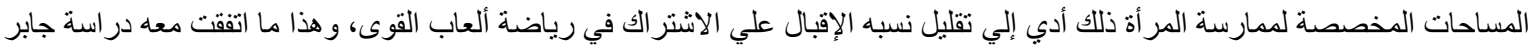

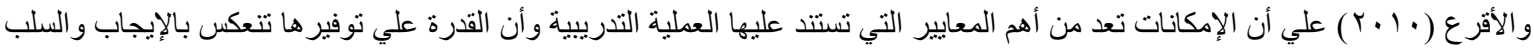
علي مستوى اللاعبين.

هذا ومن المعروف و المؤكد بأن نو افر الإمكانات الرياضية أثناء التدريب من الأمور الأساسية التي يجب تو افرها والتي بدونها لا يكون هناك

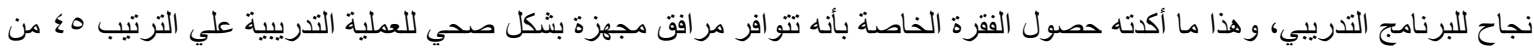

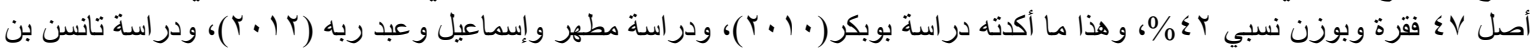

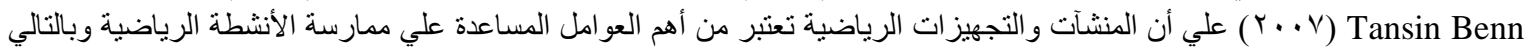
النهوض بالرياضة وجلب الأفراد نحو الممارسة الرياضية. 


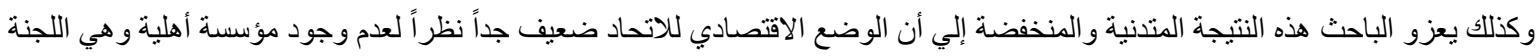

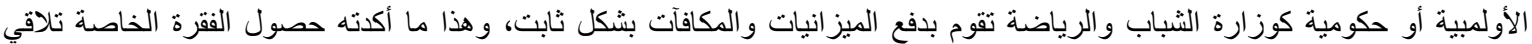

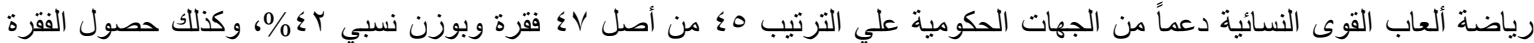

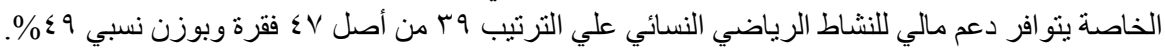

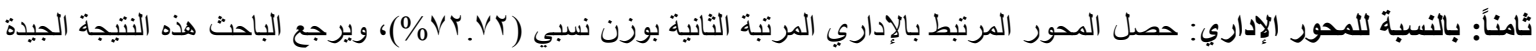

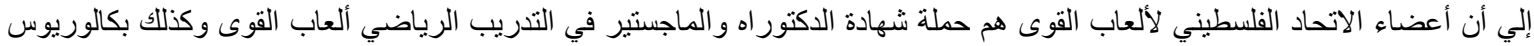

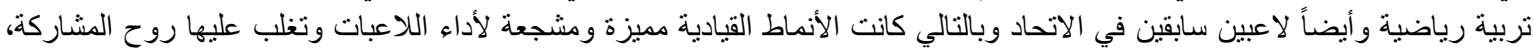

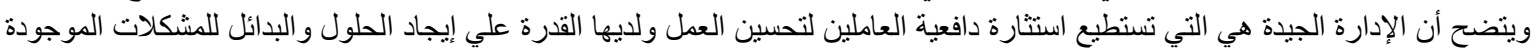

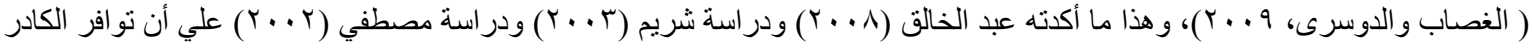

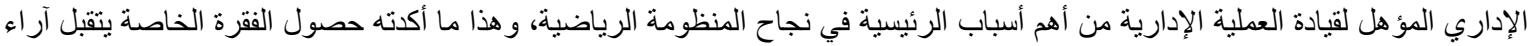

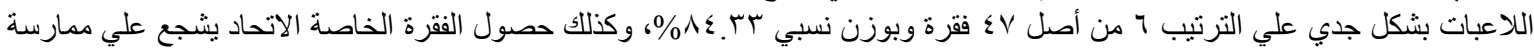

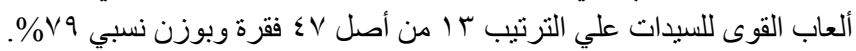

الاستخلاصات:

استناداً لنتائج البحث وفي ضوء أهداف البحث والمشكلة والتساؤلات وفي ضوء العينة والمنهج المستخدم وأدوات جمع البيانات استخلص الباحث ما يلي:

ا. مستوى تدريب ألعاب القوى للسيدات في ظل متغيرات المجتمع الفلسطيني كانت متوسطة حيث وصلت النسبة المئويـة للاستجابة

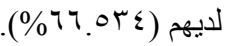

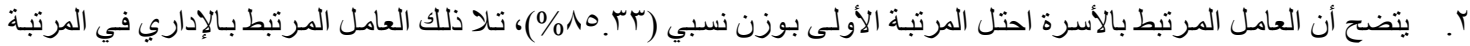

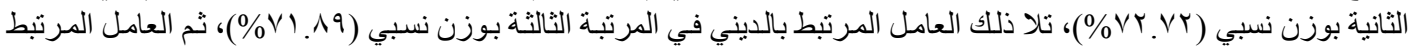

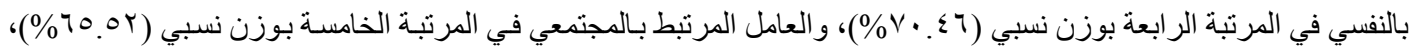

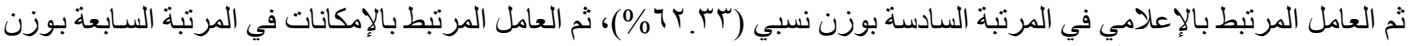

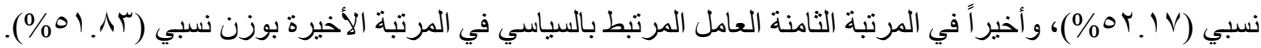

استتاداً لنتائج البحث ومن خلال أهدافه والمشكلة البحثية وتساؤلات البحث وفي ضوء العينة والهنهج المستخدم وأدوات جمع البيانات والاستخلاصات يوصي الباحث بما يلي:

ا. . ضرورة قيام وزارة الثباب والرياضة والمجلس الأعلى للثباب والرياضة بفضح وتعرية الممارسات الإسر ائيلية التي تتعمد هدم

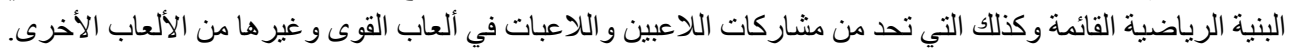
r. وقوف وزارة الثباب والرياضة والمجلس الأعلى للثباب والرياضة أمام نتائج هذه الدراسة من أجل زيادة نقاط القوة ومحاولة التغلب علي الضعف في العو امل الأخرى.

r. زيادة اهتمام اتحاد ألعاب القوى نحو الرقي بالنشاط الرياضي النسائي في فلسطين من خلال توفير الإمكانات اللازمة "المادية و والمعنوية".

؛. ضرورة اهتمام الاتحاد الفلسطيني برياضة ألعاب القوى المدرسية. •. تجهيز أماكن الممارسة الرياضية لألعاب القوى بما يو اكب التطور العلمي العالمي وتزويدها بأجهزة و أدوات قياس حديثة.

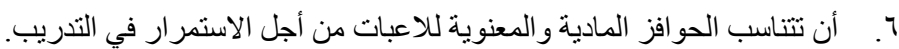
V. . ضرورة اهتمام العاملين في مجال الإعلام الرياضي بالرياضة النسائية في ألعاب القوى و إبر از انجاز اتها.

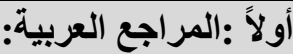

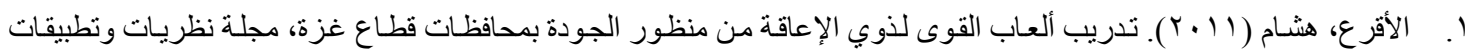

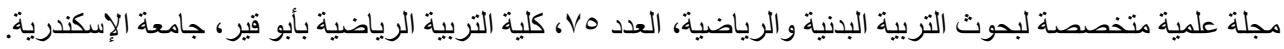




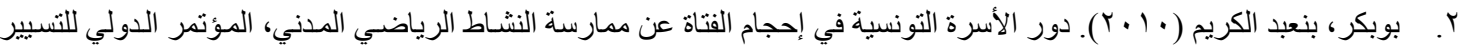
الرياضي بالجامعة التونسية، تونس.

ץ. بوبكر، بنعبد الكريم (1 · · ). ممارسة المر أة التونسية للأنشطة البدنية والرياضية وتأثير ها علي تحسين وضعها الاجتمـاعي، مجلة الكريديف، عدد ^ب، مركز البحوث و الدر اسات والتوثيق و الإعلام حول المر أة، تونس.

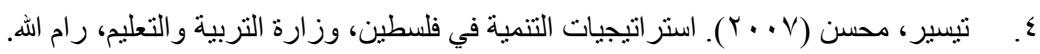

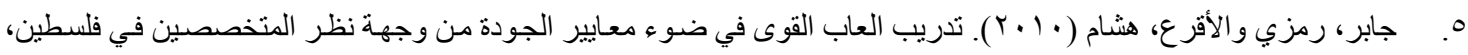
مؤتمر البحوث السابع عثر لكليات و أقسام التربية الرياضية، اس_س/ ـ ــ، كلية التربية الرياضية، جامعة تكريت. 7. شريم، زبيدة (T + . ب). اتجاهات أولياء أمور لاعبي و لاعبات التايكوندو نحو ممارسة أبنائهم للتايكوندو، المؤتمر العلمي السـادس، الجامعة الأردنية، عمان.

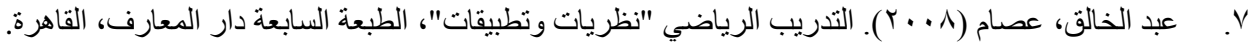

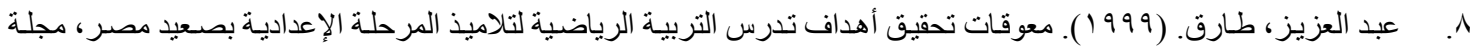
أسيوط لعلوم وفنون التربية الرياضية، الجزء الأول، العدد التاسع مارس، كلية التربية الرياضية، جامعة أسيوط . 9 9. عبد ربه، حسن وأحمد عز الدين (• ( • ). معوقات ممارسـة المر أة للنشـاط الرياضـي في الجمهوريـة اليمنية، مجلة أسيوط لعلوم وفنون التربية الرياضية، العدد • ب, الجزء الأول، كلية التربية الرياضية ، جامعة أسيوط.

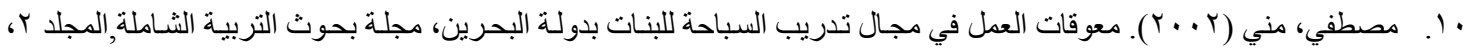
مصر

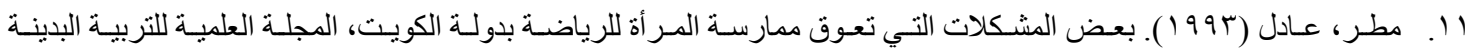
و الرياضة، العدد الخامس، كلية التربية الرياضية للبنات، جامعة الإسكندرية.

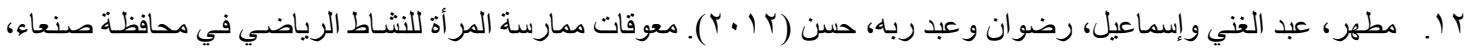
المؤتمر العلمي الدولي الأول حول الممارسة الرياضية بين المنظومة السياحية والبيئية، عــ/ ع، جامعة البويرة.

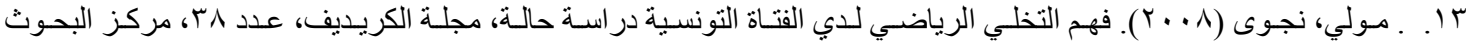
و الدراسات و التوثيق و الإعلام حول المر أة، تونس.

\section{ثُانياً :المراجع الأجنبية:}

14. Tansin Been (2007). Muslim women and sport "Rutledge studies in Physical Education and youth sport". N.J University press. U.S.A. 


\section{الملخص باللغة العربيـة}

تدريب ألعاب القوى للسيدات في ظل منغيرات المجتمع الفلسطيني. هثام علي الأقرع أستاذ التدريب الرياضي المشارك بكلية التربية البلنية والرياضة - جامعة الأقصى - فلسطين.

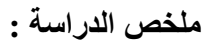

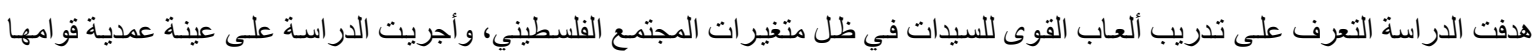

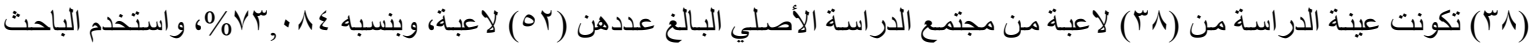
المنهج الوصفي، و أظهرت نتائج الدر اسة أن مستوى تدريب ألعاب القوى للسيدات في ظل متغير ات المجتمع الفلسطيني كانت متوسطة وبنسبة $\%(.77 .04)$

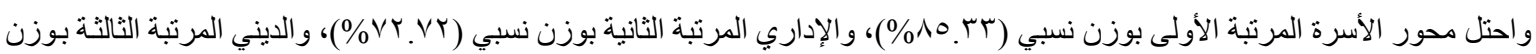

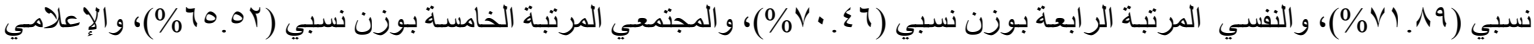

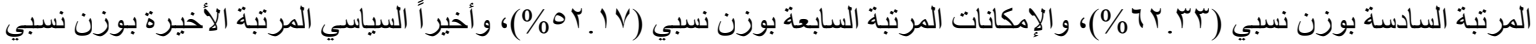


Athleties training for women under the variables of Palestinian society.

HISHAM-A-M-ALAQRA

\section{Introduction}

This study was to aimed to to identify the Athletics training for women under the variables of Palestinian society

\section{Methods}

The researcher used the descriptive approach, The study conducted on intentional Sample (38) of players.

\section{Results}

As summaries of results, showed that the level of the Athletics training for women under the variables of Palestinian society is medium (53.66\%) and the Family take the first Ranked (33.85\%), and the Administrator is the second $(72.72 \%)$, and the Religious the third $(89.71 \%)$, and the Psychological the fourth (46.70\%), and the Community the fifth (52.65\%), and the Media the sixth (33.62\%), and the potential the seventh(17.52\%), and finally the political (83.51\%).

\section{Discussion \& Conclusion}

Consequently, this study recommended to the Ministry of Youth and Sports and the Supreme Council for Youth and Sports and the erosion to expose the Israeli practices which deliberately demolish the existing sports infrastructure, as well as posts that limit the players and the players in athletics and other games other, and increase the interest of the Federation of Athletics progress toward women's sports activity in Palestine by providing the necessary resources "material and moral". 
